\title{
ESTIMATIVA DA EVAPOTRANSPIRAÇÃO EM UMA CULTURA DE ARROZ IRRIGADO UTILIZANDO DADOS METEOROLÓGICOS E DE VEGETAÇÃO
}

\author{
Vanessa de Arruda Souza ${ }^{1, *}$, Rita de Cássia Marques Alves ${ }^{1}$, \\ Eliana Veleda Klering ${ }^{1}$, Débora Regina Roberti ${ }^{2}$ \\ ${ }^{1}$ Universidade Federal do Rio Grande do Sul, Porto Alegre, RS - Brasil \\ ${ }^{2}$ Universidade Federal de Santa Maria, RS - Brasil \\ ${ }^{1}$ v.arruda.s@gmail.com
}

\begin{abstract}
This work had as objective to estimate Evapotranspiration through the PenmanMonteith, as well with the values obtained by the Eddy Covariance Method. The study area was located in the city of Cachoeira do Sul-RS, in an irrigated rice crop. The comparison between the results of the estimates of ET was satisfactory.
\end{abstract}

\section{RESUMO}

Este trabalho teve como objetivo estimar a Evapotranspiração através de PenmanMonteith, e assim comparar com os valores obtidos a partir do Método da Covariância de Vórtices Turbulentos. A área de estudo localizou-se no município de Cachoeira do Sul-RS, em uma cultura de arroz irrigado. A comparação entre os resultados das estimativas de ET foi satisfatória.

\section{INTRODUÇÃO}

A Evapotranspiração é definida pela soma da água perdida para a atmosfera a partir da superfície do solo pela evaporação, e dos tecidos das plantas através da transpiração, sendo um componente vital do ciclo da água (MU et al., 2007). O processo de evapotranspiração está diretamente relacionado com a quantidade de vapor d'água no ar, podendo alterar o regime de chuva em determinadas regiões.

Dentro deste contexto, este trabalho tem como objetivo geral estimar a evapotranspiração utilizando o Método Penman-Monteith, e assim comparar com a Evapotranspiração Real (ETr) obtida através do Método de Covariância de Vórtices turbulentos, para uma área de cultivo de arroz irrigado.

\section{MATERIAL E MÉTODOS}

A área de estudo está localizada no município de Cachoeira do Sul - RS, em uma propriedade de cultivo de arroz com aproximadamente 1000 ha. O período de estudo desta pesquisa refere-se à safra de 2010/11. Os dados meteorológicos e de Índice de Área Foliar (IAF), utilizados neste trabalho, foram coletados a partir da Rede Sulflux. Dados de 
Umidade Relativa (UR) foram utilizados da Rede INMet, a partir da estação meteorológica do município de Rio Pardo.

Conforme Allen et al. (1998), o Método Penman-Monteith é considerado pela FAO como o método padrão para o cálculo da estimativa de evapotranspiração de referência, a partir de dados meteorológicos. A equação combinada de Penman-Monteith para a estimativa da evapotranspiração utilizada neste trabalho foi:

$$
E T=f c\left(\frac{\Delta \cdot\left(R_{n}-G\right)+\rho_{a} \cdot c_{p} \cdot \frac{\left(e_{s}-e_{a}\right)}{r_{a}}}{\Delta+\gamma \cdot\left(1+\frac{r_{s}}{r_{a}}\right)} \cdot \frac{1}{\lambda \rho_{w}} \quad\left[\frac{m m}{d i a}\right]\right.
$$

Sendo: $f_{c}$ um fator de conversão de unidades e possui valor de 86,4 ; $\Delta$ representa a inclinação da curva de pressão de vapor $\left[\mathrm{kPa} .{ }^{\circ} \mathrm{C}^{-1}\right] ; R_{n}$ é o saldo de radiação diário $\left[\mathrm{W} . \mathrm{m}^{-2}\right]$; $G$ é o fluxo de calor no solo $\left[\mathrm{W} . \mathrm{m}^{-2}\right] ; \rho_{a}$ é a densidade média do ar a pressão constante $\left[\mathrm{kg} \cdot \mathrm{m}^{-3}\right] ; c_{p}$ é o calor específico do ar a pressão constante $\left[\mathrm{J}^{\mathrm{kg}} \mathrm{kg}^{-1 \mathrm{o}} \mathrm{C}^{-1}\right] ; e_{s}$ é a pressão de vapor de saturação $[\mathrm{kPa}]$; $e_{a}$ descreve a pressão atual de vapor $[\mathrm{kPa}] ; \gamma$ é a constante Psicométrica $\left[\mathrm{kPa} .{ }^{\circ} \mathrm{C}^{-1}\right] ; r_{s}$ é a resistência superficial $\left[\mathrm{s} . \mathrm{m}^{-1}\right] ; r_{a}$ é a resistência aerodinâmica

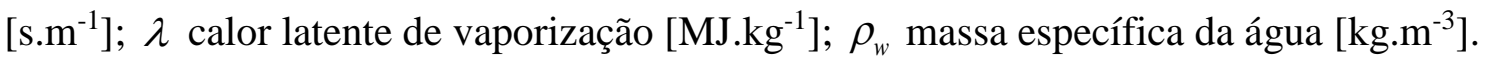

\section{RESULTADOS E DISCUSSÕES}

Para uma melhor análise do comportamento da vegetação e seu efeito sobre a evapotranspiração, foi realizado a plotagem da ET com dados de IAF coletados em campo. Através da figura 1, foi possível observar que a ET e os dados de IAF, seguem um padrão. As máximas estimativas de ET foram encontradas no período de janeiro e fevereiro, com valores entre 6 e 7 mm.dia ${ }^{-1}$.

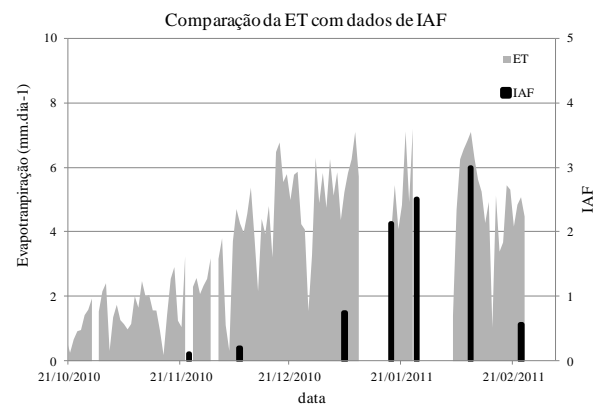

Figura 1: Comparação da evapotranspiração estimada por Penman-Monteith com dados de IAF experimentais. Período: 21 de outubro de 2010 até 04 de março de 2011.

Segundo Tabbal et al. (2002), os valores típicos de evapotranspiração da cultura de arroz nos trópicos são de 4 a $5 \mathrm{~mm} \cdot$ dia $^{-1}$, na estação chuvosa, e de 6 a 7 mm.dia ${ }^{-1}$ na estação seca. Durante o período de estudo, as chuvas foram irregulares nos volumes acumulados e na 
distribuição entre as diversas regiões do estado, causando deficiência hídrica acentuada em algumas áreas.

A comparação entre as diferentes estimativas de evapotranspiração pode ser observada na figura 2. Durante o período inicial do desenvolvimento do arroz irrigado, notamos uma diferença entre as estimativas, podendo estar ligado ao fato dos valores de IAF apresentarem pequenos valores. Verificamos também que de maneira geral a ETr superestima os valores de ET.

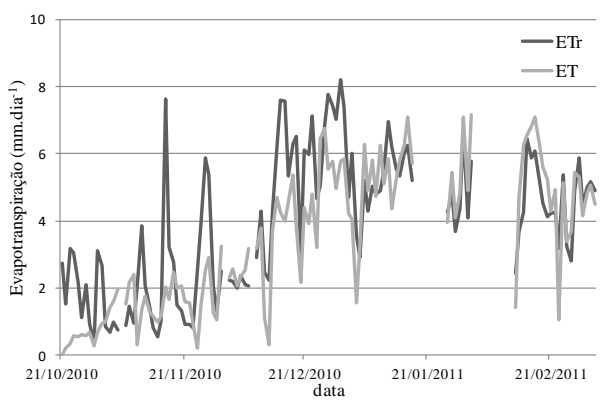

Figura 2: Comportamento das diferentes estimativas de ET. Período: 21 de outubro de 2010 até 04 de março de 2011.

\section{CONCLUSÃO}

A partir das análises realizadas neste trabalho, pode-se concluir que o comportamento temporal da estimativa de evapotranspiração e IAF seguem o mesmo padrão, devido ao desenvolvimento da cultura. A comparação entre as diferentes estimativas de evapotranspiração mostraram-se satisfatórias.

\section{AGRADECIMENTOS}

Agradecimento a Rede SulFlux e INMet aos dados fornecidos, e a FAPERGS pelo apoio financeiro.

\section{REFERÊNCIAS BIBLIOGRÁFICAS}

ALLEN, R. G.; PEREIRA, L. S.; RAES, D.; SMITH, M. Crop Evapotranspiration: Guidelines for Computing Crop Water Requirements. FAO Irrigation and Drainage Paper 56. FAO - Food and Agriculture Organization of the United Nations, Itália, 1998.

MU, Q., HEINSH, F. A., ZHAO, M., RUNNING, S. W. Development of a global evapotranspiration algorithm based on MODIS and global meteorology data. Remote Sensing Environment, v.111, p.519-536, 2007.

TABBAL, D. F.; BOUMAN,B. A. M.; BRUIYAN, S. I.;SIBAYAN, E. B.; SATTAR, M. A. On-farm strategies for reducing water input in irrigated rice: case studies in the Philippines. Agricultural Water Management, Amsterdam, v.56, n.2, p.93-112, July 2002. 